\title{
LES CORRÉLATIONS DE PERSONNE DANS L'ÉNONCÉ ORAL : ENJEUX ÉNONCIATIFS ET PROSODIQUES
}

\author{
Amidou SANOGO \\ Université Félix Houphouët-Boigny de Cocody-Abidijan, Côte d'Ivoire \\ sanogo.amidou@univ-fhb.edu.ci \\ Article reçu le 22 mars 2016 | révisé depuis le 15 avril 2016 | accepté le 23 juin 2016
}

\begin{abstract}
RÉSUMÉ. Selon Benveniste (1966), l'espace énonciatif se répartit entre personnes (Je/Tu) et non-personne (Il). On peut citer, entre autres critères de détermination, les oppositions de nombre et les propriétés syntaxiques et les phénomènes suprasegmentaux tels que le ton et l'intonation. Celle-ci correspond à la variation de la hauteur de la voix au cours de l'énonciation. L'étude aborde des énoncés de type oral avec une construction segmentée par une pause virgulaire. Cette segmentation comporte des implications prosodiques et distributionnelles qui fondent nos préoccupations. Dès lors, quelles peuvent être les incidences de ces phénomènes suprasegmentaux sur les corrélations de personne $J e / T u$ et $J e, T u / I l$. L'étude a pour objectif de montrer la fonction différentielle de certains prosodèmes comme le ton, l'intonation, l'accent, etc. elle revêt l'intérêt de démontrer les limites de la dichotomie personne \# non personne. Notre hypothèse est que les phénomènes prosodiques contribuent à la cohésion des instances énonciatives à travers la catégorie de la personne. L'étude se propose d'examiner les schémas intonatifs et d'en dégager les propriétés sous l'angle de la personne grammaticale.
\end{abstract}

Mots-clés: distributionnel, énonciation, intonation, personne, prosodie.

\begin{abstract}
Benveniste's (1966) theory underscores that the enunciation's space is divided between the persons (I / you) and non-person (It, he). These include, among other criterias, the opposition of numbers (singular/plural) and the syntactic properties (substitution, inversion...), which ones go along with some suprasegmental phenomena such as intonation. The intonation is the variation of the pitch of the voice when producing utterances. Therefore, what are the implications of these suprasegmentals phenomena on person correlations between I / You and I, You / It. The present contribution intends to highlight the differential properties of some prosodemes like tone, intonation and stress, etc., in the understanding of the dichotomy person \# non-person. We hypothesize that the suprasegmental realizations help to the cohesion of the different levels of enunciation through the category of the person. In this study, we want to go through the intonation patterns and identify their properties on the perspective of the grammatical person.
\end{abstract}

Keywords: distributional, enunciation, intonation, person, prosody. 


\section{INTRODUCTION}

Depuis l'enseignement de Benveniste (1966), la catégorie de la personne connaît une double opposition traduite, d'une part, par la double corrélation: celle de subjectivité (Je/Tu) et celle de personne (Je, $\mathrm{Tu} / \mathrm{Il})$. Les critères formels de la dichotomie personne \# non personne sont les oppositions de nombre (masculin/singulier) et les propriétés syntaxiques (substitution, inversion...). Cette double opposition repose sur la division de l'espace énonciatif entre personne (Je/Tu) et non-personne (Il). Les critères de différentiation s'accompagnent de phénomènes suprasegmentaux, en l'occurrence l'intonation. Celle-ci correspond à la variation de la hauteur de la voix au cours de l'énonciation.

Dès lors, il convient de questionner les corrélations entre les indices de personne à travers cet aspect de la prosodie. Quelles sont les implications prosodiques et distributionnelles de cette corrélation?

L'étude vise à montrer la fonction différentielle de certains prosodèmes comme le ton, l'intonation, l'accent, etc., dans la compréhension de la dichotomie personne \# non personne.

Notre hypothèse est que les phénomènes prosodiques contribuent à la neutralisation de l'opposition personne \# non personne et, partant à la cohésion linguistique des instances énonciatives.

L'étude a recours à un double modèle théorique énonciatif et structuraliste de façon plus large. Elle examine d'abord les procédés syntaxiques de dislocation d'un terme de l'énoncé et les opérations linguistiques correspondantes dont la thématisation et l'apostrophe; ensuite, elle décrit la relation entre les schémas intonatifs dans les énoncés de type oral et ces opérations; enfin, elle analyse les propriétés prosodiques mises en œuvre dans ces énoncés pour assurer la cohésion entre personne \# non personne.

\section{LA PAUSE INTONATION DANS L'OPÉRATION LINGUISTIQUE DE THÉMATISATION}

Dans la structure $\mathbf{N}_{0} \mathbf{V} \mathbf{N}_{\mathbf{1}} \mathbf{X}$, le détachement de $\mathbf{N}_{0}$ consiste en l'extraposition du sujet $\mathbf{N}_{0}$ hors du prédicat verbal ; ce qui a pour effet l'emphatisation. On ne retiendra les termes d' "emphase », et de " mise en relief » qui désignent, à la fois, un procédé de thématisation (par le détachement par exemple) et un procédé de rhématisation (par le clivage par exemple). Comme le dit Le Querler (1998, p.114), ces deux termes placent le commentaire linguistique du côté du locuteur. Dès lors, la présente étude privilégie la posture énonciative du locuteur qui marque son énoncé de façon particulière. Il s'agit, ici, de la dislocation appelée également détachement ou extraction. A l'instar de Le Querler (1998, p.114), nous utilisons le terme de dislocation au sens de procédé syntaxique disjoignant de son terme recteur un constituant de la phrase.

Dans l'énoncé, la dislocation s'effectue au moyen de la pause-intonation symbolisée par le signe du dièse (\#) (Rossi, 1990). Ce procédé aboutit à un schéma intonatif tel que défini par Morel (1992b, p.61-74). Il est composé du segment modal (qui définit la nature de la modalité de l'énoncé), du thème (ce qu'on dit) et du rhème (ce dont on parle). Ainsi formé, l'indice de personne n'apparaît pas de la même manière dans les groupes rythmiques correspondant au schéma mélodique constitué par la protase et l'apodose. Ainsi, selon Grégoire (1971), "le mouvement rythmique et mélodique de la phrase est $[\ldots]$, à la fois, le produit et l'indispensable actualisateur des autres structures de l'énoncé" (p.117).

L'étude part de la phrase ou «contourème» pour en décrire l'unité mélodique ou «intonème ». Ainsi, le corpus, ci-devant, se prête à cette analyse par son oralité. Il est constitué d'énoncés de conversation libre échappant aux normes canoniques de la phrase noyau même si l'on peut les réduire à ce schéma irréductible $(\mathrm{P}$ : $\mathrm{SN}+\mathrm{SV})$. Au niveau suprasegmental, ces types d'énoncé sont dominés par des émotions qui confèrent à l'énoncé une structure particulière. Ainsi, le roman La vie et demie de Sony présente les propos d'un 
tortionnaire empreints de supplications et de colère devant la résistance de sa victime :

E1 : Alors, quelle mort veux-tu mourir, Martial?

(Sony, 1979, p. 13),

E2 : Sois raisonnable, Martial, et dismoi quelle mort tu veux mourir ?

(Sony, 1979, p.12-15) ;

E3 : Enfin, Martial, sois raisonnable.

(Sony, 1979, p.14);

E4 : Enfin, Martial! Combien de fois veux-tu que je te tue?

(Sony, 1979, p.19).

Le corpus repose sur des suites de conversations identifiables aux connecteurs «alors» E1 et «enfin» E3, E4; il place le commentaire linguistique sur le plan allocutif avec des énoncés de type oral.

Dans ces énoncés, on relève la variation de la ponctuation: la ponctuation haute (point d'interrogation, point d'exclamation) et la ponctuation basse (virgule, point) dans un même énoncé (E1, E2, E3, et E4). La pause virgulaire correspond au marquage syntaxique de la thématisation d'un actant de la phrase. On parlera ici de dislocation (à gauche ou à droite), la position structurelle de l'élément disloqué ne correspondant pas obligatoirement aux mêmes types de thématisation.

- Aussi peut-on observer trois structures syntaxiques différentes dans le corpus :

- dislocation du complément d'objet direct «Martial» à gauche avec pronominalisation

- par le constituant «te » (E4) ;

- dislocation à gauche du terme «Martial» sans pronominalisation (E2, E3) ;

- dislocation à droite de «Martial» sans pronominalisation (E1) ;

Les structures syntaxiques en présence sont quasi identiques pour permettre de ressortir un certain nombre de régularités de ces énoncés.

Ainsi, les structures syntaxiques débouchent sur les trois groupes rythmiques (3 g.r.) suivants :

E'1:/alors / quelle mort veux-tu mourir / Martial //

E'2 : /sois raisonnable/ Martial/ et dismoi quelle mort tu veux mourir //
E'3 : / enfin / Martial / sois raisonnable //

E'4 : / enfin/ Martial // combien de fois veux-tu que je te tue //

En outre, ces dislocations correspondent à des emphases ou des mises en relief décrivant tous, des procédés de thématisation. Celle-ci correspond à une opération de topicalisation à gauche du prédicat verbal (E2, E3 et E4) ou à un rejet à droite du nœud verbal (E1).

La description de la modalité emphatique permet $d$ 'isoler les termes emphatisés en liaison avec un indice de sujet dont il est le référé discursif : Martial (tu) E'1, $E^{\prime} 2, E^{\prime} 4$ et $E^{\prime} 3$. Dans $E^{\prime} 3$, ce référé discursif se réalise par la marque zéro de la modalité impérative.

L'opération de détachement subséquent s'effectue en finale ( $\left.E^{\prime} 1\right)$ en médiane $\left(E^{\prime} 2\right)$ et à l'initiale après le connecteur «enfin » (E'3 et $\left.E^{\prime} 4\right)$. Le lieu du détachement correspond à une unité mélodique que l'étude va déterminer.

Parmi les dix unités mélodiques de base du français telles que déterminées par Delattre (1966), ces énoncés présentent des unités suivantes correspondant aux intonations appliquées aux phrases. Celles-ci sont simples ou composées dans leurs structures. Elles interviennent concomitamment dans la même chaîne phrastique.

Ces énoncés recouvrent les unités mélodiques liées à des modalités d'interrogation qui peuvent être réparties en deux catégories : l'intonation d'interrogation et l'intonation de question.

\section{Intonation de question-intonation d'interrogation}

L'intonation de question accompagne l'interrogation totale qui demande une réponse par «oui» ou par «non». Les énoncés ci-dessous ne correspondant pas à ces critères, il y a lieu d'exploiter l'intonation d'interrogation.

Ainsi, l'interrogation est l'intonation propre aux énoncés interrogatifs partiels introduits par un monème interrogatif 
comme «quelle» $E^{\prime} 1$ et $E^{\prime} 2$, et «combien» $\mathrm{E}^{\prime} 4$.

L'intonation d'interrogation est rattachée aux structures syntaxiques, ciaprès, toujours avec trois groupes rythmiques (3 g.r.) :

a) Syntaxe $=$ morphème interrogatif + inversion du sujet.

E'1 : /alors / quelle mort veux-tu mourir / Martial // (3 g.r.)

Unités mélodiques: $\mathrm{Cm}+\mathrm{Q}+\mathrm{Ph}+\mathrm{Im}$

E'4: / enfin/ Martial // combien de fois veux-tu / que je te tue // (3g.r.)

Unités mélodiques: $\mathrm{Cm}+\mathrm{Cm}+\mathrm{Q}+\mathrm{Ph}+\mathrm{Im}$

Dans ces réécritures, la parenthèse haute (Ph) suit obligatoirement une intonation descendante après une intonation de finalité, d'interrogation (Q), d'ordre ou d'exclamation.

b) Syntaxe $=$ énonciative + morphème interrogatif.

Ici, apparaissent concomitamment les intonations d'ordre, d'implication (Im) et de parenthèse basse $(\mathrm{Pb})$. Cette dernière est constituée de tons ayant la même hauteur. La parenthèse basse suit forcément une ligne mélodique descendante par opposition à la parenthèse haute qui est précédée d'une ligne mélodique haute.

E'2 : /sois raisonnable/ Martial/ et dis-moi quelle mort tu veux mourir // Unités mélodiques: Ordre $+\mathrm{Im}+\mathrm{Cm}+$ Ordre $+Q$ $+P h$

Dans la syntaxe énonciative, l'interrogation n'est pas syntaxiquement marquée; elle est perceptible au niveau prosodique par la seule intonation montante. Quant à l'impératif, elle est marquée par l'intonation de parenthèse basse $(\mathrm{Pb})$.

\section{Intonation de continuation-Intonation exclamative}

L'intonation de continuation est l'unité mélodique composée de continuation majeure $(\mathrm{CM})$ et de la continuation mineure (Cm) qui affectent les groupes rythmiques dans la phrase. Grégoire (1971, p. 117) dit que l'intonation $\mathrm{Cm}$, "dans une phrase à $n$ groupes rythmiques», porte sur «tous les premiers groupes jusqu'à $n-2$ compris. Le groupe n-1 aura une intonation de CM...». Elle revêt un contour mélodique convexe allant $\mathrm{du}$ niveau 2 au niveau 4 . Elles interviennent secondairement, dans les énoncés, auprès d'autres intonations dominantes comme :

- l'intonation interrogative

E'1 : /alors / quelle mort veux-tu mourir / Martial // (3 g.r.)

Unités mélodiques: $\mathrm{Cm}+\mathrm{Q}+\mathrm{Ph}+\mathrm{Im}$

$E^{\prime} 4 b$ : /combien de fois veux-tu / que je te tue //

Unités mélodiques: $\mathrm{Q}+\mathrm{Ph}+\mathrm{Im}$

- l'intonation impérative (Ordre)

E'3 : / enfin / Martial / sois raisonnable //

Unités mélodiques: $\mathrm{Cm}+\mathrm{CM}+$ Ordre $+\mathrm{Pb}$

$+\mathrm{Im}$

E'2a : /sois raisonnable / Martial//

Unités mélodiques: Ordre $+\mathrm{Pb}+\mathrm{Im}$

$\mathrm{E}^{\prime} 2 \mathrm{~b}$ : / dis-moi / quelle mort tu veux mourir //

Unités mélodiques: Ordre $+Q+P h+I m$

$L$ 'ordre représentant la valeur de base de l'intonation impérative s'accompagne de l'intonation d'implication (Im) sousentendant une supplication. Celle-ci constitue la valeur pragmatique de l'interrogation et de l'impératif. Dans le contexte de l'interaction langagière entre le tortionnaire et sa victime, l'interrogation ne saurait être le support référentiel d'une question. C'est une interrogation délibérative appelée également fausse interrogation au sens de Grevisse (1991, p.635) ou oratoire qui suppose une requête et même une colère devant la résistance surréaliste du supplicié. Elle sert plutôt à exprimer la valeur d'une relation de co-énonciation fondée sur la rupture entre les deux acteurs qui devrait se solder par la mort de Martial. Elle souligne la contrariété de l'évidence. De même, l'impératif exprime une valeur pragmatique de supplication et de prière. L'ordre n'est pas formel. C'est un pseudo-ordre avec un ton bas.

En outre, on note une intonation rattachée aux précédentes: l'intonation de parenthèse constituée de tons de même hauteur. On distingue la parenthèse haute, avec une ligne mélodique montante après une question et la parenthèse base $(\mathrm{Pb})$ avec 
une ligne mélodique descendante qui intervient après l'interrogation, l'exclamation ou l'ordre.

Dans les deux cas de figure, les énoncés n'ont aucun pouvoir interrogatif ni impératif. A leurs modalités de base, s'ajoutent des intonations supplémentaires qui modifient la courbe mélodique de l'énoncé et font valoir des nuances.

- l'intonation exclamative (E)

Syntaxe $=$ adverbe + exclamation

E'4a : / enfin / Martial //

Unités mélodiques: $\mathrm{Cm}+\mathrm{E}$

L'intonation exclamative contribue à donner la réaction d'un sujet locuteur en traduisant ses sentiments. Elle est marquée par un point d'exclamation. Ici, l'on perçoit un sentiment d'exaspération qui doit faire monter l'intonation plus haut. L'intonation exclamative est une nuance qui affecte d'autres intonations telles que celle de continuation. Elle a donc une fonction expressive.

On retient que dans les énoncés, la « dislocature » (Damourette \& Pichon, 1930, T3, p. 424), ou construction segmentée (Bally, 1965, p.62), est matérialisée mélodiquement par une intonation montante (E2, E3, et E4) ; l'énoncé E4 présente l'intonation accompagnée d'un accent d'intensité. Cela est remarquable avec l'exclamation, signe de l'émotion éprouvée par le locuteur. Il s'agit d'un accent d'insistance, ou accent expressif, qui procède de la même modalité de mise en évidence que l'emphatisation. L'accent tonique préexistant à celui-là, est inhérent aux autres énoncés du corpus. Il contribue au rythme de la parole.

Les phénomènes prosodiques n'ont pas seulement une fonction purement expressive; ils jouent également un rôle important dans l'échange linguistique en guidant l'interlocuteur dans la compréhension efficace du message. Les unités mélodiques sous-tendant les énoncés obéissent à des schémas intonatifs.

\section{STRUCTURE SYNTAXIQUE ET INTONATION DANS LES ÉNONCÉS ORAUX}

Le schéma intonatif des énoncés se compose essentiellement de deux ensembles dont le premier est le repère (alors/ enfin/ dis-moi) et le second, le repéré (quelle mort veux-tu mourir, Martial ?/ sois raisonnable, Martial/ quelle mort tu veux mourir/ Martial! Combien de fois veux-tu que je te tue?).

\section{Intonation dans l'ensemble repéré}

Théoriquement, dans l'ensemble repéré, l'ordre thème -prédicat, correspond aux intonations de continuation (C) et de finalité (F). Ceci concerne les énoncés de type déclaratif avec le schéma canonique Sujet + verbe + objet. Mais, dans les constructions interrogatives (E'1, E'2b, E'4), l'ordre canonique est inversé : l'objet direct est détaché à l'initiale, après l'adverbe « alors ", la locution verbale «dis-moi » et d'autres éléments de l'ensemble repère. Ils constituent cependant le thème de cette séquence de l'énoncé global avec des variations d'intonations selon les modalités d'énonciation :

E'1:/alors / quelle mort /veux-tu mourir / Martial //

Unités mélodiques: $\mathrm{Cm}+\mathrm{Q}+\mathrm{Ph}+\mathrm{Im}$

Les variations concernent l'intonation de parenthèse basse $(\mathrm{Pb})$ qui intervient après la question et celle de l'implication (Im) qui nuance l'interrogation.

Avec E'2, on observe un double schéma intonatif coordonné par la conjonction « et »:

E'2a : / Sois raisonnable/ Martial//

$E^{\prime} 2 b$ : / dis-moi / quelle mort tu veux mourir //

Dans E'2a, le thème est postposé au prédicat. Ce qui correspond aux unités mélodiques suivantes :

E'2a : / Sois raisonnable/ Martial//

Unités mélodiques : Ordre $+\mathrm{Pb}+\mathrm{Im}$

Dans ces deux premiers énoncés, le nom détaché «Martial » est caractérisé par un ton bas à la chute de l'énoncé; il est faiblement thématisé (Sabio, 2006). Il s'agit d'un cas de « rejet » par opposition à la «topicalisation » qui décrit le détachement à gauche. Quant à $\mathrm{E}^{\prime} 2 \mathrm{~b}, \mathrm{l}^{\prime}$ ordre thème -prédicat est respecté.

$\mathrm{E}^{\prime} \mathrm{b} \mathrm{b}:$ / quelle mort tu veux mourir //

Unités mélodiques : $Q+P h+I m$ 
E'3 et E'4 présentent un schéma intonatif avec un ligateur (enfin), un thème « Martial» et un prédicat « sois raisonnable » :

\section{E'3 : /Enfin / Martial/ sois raisonnable // \\ Unités mélodiques: $\mathrm{Cm}+\mathrm{CM}+$ Ordre + $\mathrm{Pb}$ \\ Cette structuration est équivalente à} celle de $\mathrm{E}^{\prime} 4$

E'4 : / Enfin/ Martial // Combien de fois veux-tu que je te tue //

Unités mélodiques: $\mathrm{Cm}+\mathrm{Cm}+\mathrm{Q}+\mathrm{Ph}+$ Im

Dans ces deux derniers exemples, le nom détaché «Martial » est affecté d'un ton moyen dans la partie médiane de l'énoncé ; la thématisation y est forte (Sabio, 2006) avec le marqueur thématique «enfin ». L'unité mélodique a une valeur de continuation dite mineure. Il s'agit d'un cas de «topicalisation » qui décrit le détachement à gauche. Les deux opérations ainsi décrites conviennent à l'opération linguistique de l'apostrophe que Nølke (1997, p.288) distingue de la dislocation. On note cependant le lien référentiel de «Martial» avec les pronoms clitiques «tu», «te» présents dans le prédicat verbal.

Ces énoncés présentent des variations dues aux intonations de parenthèse haute (Ph) et d'implication (Im). Ces intonations traduisent les émotions du Guide dans le roman plus qu'elles n'expriment des modalités d'énonciation formelles (interrogation, ordre).

\section{Intonation dans l'ensemble repère}

L'ensemble repère correspond au thème traditionnel défini par Morel (1992b). Il définit l'orientation de l'allocution (adresse à l'allocutaire) et constitue le cadre où s'inscrit la relation prédicative. Ainsi, sur le plan syntaxique, le constituant post-verbal, l'objet direct (quelle mort), est détaché du verbe recteur (mourir) ; il en est de même du circonstant (combien de fois) dans son rapport avec le verbe (tue). Ces détachements décrivent des opérations linguistiques fondées sur le procédé syntaxique de la dislocation. Pour Morel (1992a, p.34), les dislocations sans pronominalisation sont un type particulier de thématisation qu'elle appelle le « cadrage ».

En outre, on relève un autre élément qui permet de construire un cadre sans rien y inscrire $\mathrm{du}$ point de vue sémanticoréférentiel. C'est le paradigme des marqueurs thématiques (Lagae, 2011) avec l'adverbe «enfin» et la locution verbale « dis-moi » appelés ligateurs (lig.) (Morel, 1992b).

Cet emploi pragmatique de «enfin» peut être rendu par «voyons». Il a une valeur de réprobation. Quant à la locution verbale «dis-moi », elle est à la deuxième personne de l'impératif. D'où l'unité mélodique d'ordre qui l'affecte. Ces deux connecteurs servent à établir le lien avec un contexte antérieur gauche. En tant que ligateurs, ils spécifient le positionnement du locuteur par rapport à son interlocuteur. C'est une sorte de captatio benevolentiae (recherche de la bienveillance et de l'adhésion de l'auditoire) qui définit la colocution et sert de préface à la co-énonciation.

Les structures des différents énoncés sont représentées dans le tableau cidessous :

Tableau 1. Structuration des énoncés oraux

\begin{tabular}{|c|c|c|c|c|}
\hline & Lig. & Modalité & Thème (repère) & Rhème (repéré) \\
\hline$E^{\prime} 1$ & alors & & Martial & quelle mort veux-tu mourir \\
\hline$E^{\prime} 2 a$ & & & Martial & sois raisonnable \\
\hline$E^{\prime} 2 b$ & dis-moi & & & quelle mort tu veux mourir \\
\hline$E^{\prime} 3$ & Enfin & & Martial & sois raisonnable \\
\hline$E^{\prime} 4$ & Enfin & & Martial & Combien de fois veux-tu que je te tue \\
\hline
\end{tabular}


Les ensembles repère et repéré sont les résultantes de la dislocation intervenue dans la chaîne phrastique. Le schéma intonatif qui la représente fait apparaître des constituants de l'énoncé oral surmonté de prosodèmes. Ces éléments suprasegmentaux comportent des intérêts suivant les distributions des unités personnelles qui réfèrent à des instances énonciatives.

\section{Enjeux énonciatifs de la dislocature}

La structuration de l'énoncé oral revêt l'intérêt d'analyser la dislocature entre le thème (repère) et le rhème (repéré). Les énoncés E2, E3 et E4 relèvent du plan locutoire où le locuteur est prééminent (Damourette et Pichon, 1968: 308) avec l'exclamation nominale (Martial! (E4)), le vocatif (Martial (E2, E3)), et l'impératif (sois raisonnable (E3)). Le plan allocutoire concerne l'énoncé E1 avec l'interrogation où le locuteur cède son rôle à l'allocutaire qui doit répondre à la préoccupation en suspens. Mais quand on se réfère à l'objet de ces propos, c'est le plan délocutoire qui apparaît pour tous ces énoncés. On retrouve la position de Guillaume (1991, p 114) selon qui, la personne délocutive n'est absente d'aucune personne des trois personnes puisqu'il est toujours parlé d'une personne, laquelle, dans le cas de la personne locutive, est celle-là même qui parle, et dans le cas de la personne allocutive, celle-là même à qui l'on parle.

Avec lui, on dira que "sous toute personne, il $\mathrm{y}$ a une troisième personne" (Guillaume, p.114). Ainsi, il n'empêche qu'à travers les modalités exclamative, emphatique (vocatif), d'une part, et l'interrogation, d'autre part, ces énoncés du plan délocutoire présentent des marques de l'organisation des plans locutoire et allocutoire. Ce plateau intonatif est donc propice à l'apparition des indices locutoires, allocutoires et délocutoires. Dès lors, il est possible d'établir une dichotomie tonémique (différence de ton) selon que l'unité personnelle apparaît dans le repère, l'indice de modalité, ou dans le segment repéré.

De façon pratique, quand l'indice personnel surgit dans le segment modal, il y a deux faits remarquables. Observons les énoncés ci-dessous :

E1 : Alors, quelle mort veux-tu mourir, toi, Martial.

E2 : Sois raisonnable, toi, Martial, et dis-moi quelle mort tu veux mourir? (Sony, 1979, p.12-15)

E3 : Enfin, toi, Martial, sois raisonnable. (Sony, 1979, p. 14);

E4 : Enfin, toi, Martial! Combien de fois veux-tu que je te tue? » (Sony, 1979, p.19).

Appliquons les transformations de déplacement sur le segment modal :

[Transf. Déplacement] Alors, toi, Martial, quelle mort veux-tu mourir.

Sois raisonnable, toi, Martial, et dismoi quelle mort tu veux mourir?

Enfin, toi, Martial, sois raisonnable. (p. 14);

Enfin, toi, Martial! Combien de fois veux-tu que je te tue? ».

D'abord, l'indice personnel prend la forme tonique et permet de préciser la valeur de la co-énonciation. Ce pronom (toi) devient par conséquent le support de validation de la prédication qui sera réalisée dans le rhème suivant (quelle mort veux-tu mourir / dismoi quelle mort tu veux mourir/ sois raisonnable / combien de fois veux-tu que je te tue ?). Ensuite, le pronom, qui devient un indice de sujet (tu) incorporé au noyau verbal, va constituer l'origine des constructions référentielles introduites dans le thème. Ce qui permet de sceller les liens intonatifs et syntaxiques entre les indices de modalité et le thème.

Du point de vue morphosyntaxique, le nominal «Martial» extraposé est en corrélation avec le pronom «Toi »; les deux unités ont pour variante contextuelle le flexif personnel «tu». On retrouve, ici, une particularité linguistique du vocatif qui fait passer le nominal de la troisième à la deuxième personne. Ici, le vocatif est une forme d'interpellation de l'allocutaire. Le tour emphatique se renforce avec le support du pronom tonique.

En conséquence, l'intonation qui accompagne l'énoncé oral de modalité emphatique a une fonction de cohésion en ce sens qu'elle établit une passerelle entre la 
personne et la non personne. Cet élément de prosodie est la marque d'opérations discursives diversement désignées par les termes «interpellation» et «vocatif». L'intonation est syntaxiquement reconnaissable à la construction segmentée de l'énoncé à l'aide de la pause virgulaire. Ceci correspond à l'organisation du plan énonciatif en deux plans. L'énoncé présente alors une dislocature, en l'occurrence, avec l'unité en position de thème, qui relève du plan délocutif. Quant au second plan, il est allocutif avec la marque de la deuxième personne incorporée au verbe sous forme de désinence ou de flexif personnel.

\section{CONCLUSION}

L'analyse mélodique des énoncés révèle des ressources prosodiques de l'énonciation orale. La structure mélodique est plus expressive que l'agencement simple des formes. Les opérations de détachement (théorie syntaxique) ou de thématisation (logique formelle) font intervenir des phénomènes supra segmentaux. Elles aboutissent à la mise en saillance d'une unité de la chaîne parlée. Ce fait linguistique concerne principalement la catégorie de la personne repérable selon des critères distributionnels.

L'étude a permis de redécouvrir une autre dimension des travaux de Benveniste (1966) sur la double corrélation de subjectivité $(\mathrm{Je} / \mathrm{Tu})$ et de personnalité $(\mathrm{Je}, \mathrm{Tu} /$ $I l)$. Cette réflexion établit une ligne de démarcation nette entre le couple Je / Tu et Il. Mais à travers l'analyse du schéma intonatif, il est apparu une passerelle entre les deux de corrélations établissant ainsi, des similitudes entre la personne du couple Je/tu et la nonpersonne Il. En d'autres termes, il faut mentionner que dans certaines circonstances d'emploi, liées aux phénomènes suprasegmentaux, les positions $\mathrm{Je}, \mathrm{Tu}$ et $\mathrm{Il}$ génèrent des propriétés linguistiques visant à rétablir l'unité de la catégorie de la personne.

\section{REMERCIEMENTS}

Je remercie l'équipe du laboratoire des théories et modèles linguistiques (ltml), pour sa contribution, et la direction de l'Université
Félix Houphouët-Boigny d'Abidjan pour son appui financier.

\section{RÉFÉRENCES}

Bally, C. (1965). Linguistique générale et linguistique française. (IVe éd., revue et corrigée). Berne : Francke.

Benveniste, E. (1966). Problèmes de linguistique générale 1. Paris : Gallimard

Damourette, J. et Pichon, E. [1930]. Des mots à la pensée, Essai de grammaire de la langue française. Paris : d' Artrey.

Delattre, P. (1966). Les dix Intonations de base du francais. The French Review. 40, (1), 1-14.

Gregoire, H.C. (1971). L'intonation de la phrase française. Etudes préliminaires à un manuel d'exercices structuraux. Annales de l'université d'Abidjan, 4 (1), 115-141.

Grevisse, M. (1991). Le bon usage. Paris, Duculot.

Guillaume, G. (1991). Leçons de linguistique. (1943-1944 A, vol. X). Québec : PUL.

Lagae, V. (2011) Le paradigme des marqueurs thématiques en français: essai de typologie. In E. Comes \& Miculescu, S. (éds), La construction d'un paradigme - Actes du XVIIIe Séminaire de Didactique Universitaire Constanta 2010. Cluj : Editura Echinox, p 53-74.

Le Querler, N. (1998). Le marquage syntaxique de la de l'objet dans La Pluie d'été de Marguerite Duras. Cahiers de Praxématique, 30, 113-131. Repéré http://praxematique.revues.org/2759

Morel, M-A. (1992a). Intonation et thématisation. L'Information Grammaticale, 54, 26-30.

Morel, M-A. (1992b). L'opposition thèmerhème dans la structuration des dialogues oraux. Journal of french Language Studies, 2 (1), 61-74.

Nølke, H. (1994). Linguistique modulaire : de la forme au sens, Louvain-Paris: Peeters-S. I. G.

Nølke, H. (1997). Note sur la dislocation du sujet: thématisation ou focalisation?. Dans G. Kleiber \& M. Riegel (dir.), Les formes $d u$ sens. Louvain-la-Neuve: Duculot. 
Rossi, M. (1990). Ordre, organisation et intonation. Dans AA.VV (dir.). Scritti in onore di Lucio Croatto. Padova : Centro di Studio per li recerche di Fonetica del C.N.R.
Sabio, F. (2006). L'antéposition des compléments dans le français contemporain : L'exemple des objets directs. Lingvisticae Investigationes, 29(1), 173-182.

Sony. L.T. (1979). La vie et demie. Paris: Editions $\mathrm{du}$

Seuil 